\title{
IS IT POSSIBLE TO RECOVER THE CORRECT THEORY OF GRAVITY FROM COSMOLOGY?*
}

\author{
LESZEK M. SOKOŁOWSKI \\ Astronomical Observatory, Jagiellonian University \\ Orla 171, 30-244 Kraków, Poland \\ lech.sokolowski@uj.edu.pl \\ (Received October 14, 2015)
}

\begin{abstract}
We briefly present the concept of determining the true theory of gravitation using cosmological observations and criticize it.
\end{abstract}

DOI:10.5506/APhysPolB.46.2335

PACS numbers: 04.50.Kd, 98.80.Jk

\section{Introduction}

To the particle physicist the title question may seem bizarre, however, it concerns a significant direction of fundamental research to which a wealth of works have been devoted. Actually, the idea of connecting cosmology to search for the correct theory of gravitation comes from the leading cosmological discovery that the luminosity distance to the type Ia supernovae as a function of their redshift $z, d_{L}(z, \mathrm{obs})$ is larger than their distance computed within the Canonical Cosmological Model, $d_{L}(z, \mathrm{CCM})$. By the Canonical Model, we mean the standard Friedmann-Lemaitre dynamical model in the homogeneous and isotropic Robertson-Walker $(\mathrm{R}-\mathrm{W})$ spacetime filled with ordinary matter (including dark matter) and for the cosmological constant $\Lambda=0$. The inequality $d_{L}(z, \mathrm{obs})>d_{L}(z, \mathrm{CCM})$ is usually interpreted as an effect of an accelerating expansion of the universe, that is, the cosmic scale factor $a(t)$ grows faster and faster, $\ddot{a} \equiv\left(d^{2} / d t^{2}\right) a>0$; if true, the discovery is important since the acceleration is excluded in the Canonical Model. We wish to emphasize that the acceleration is merely an interpretation of the observations and it is not a direct and unambiguous outcome of pure measurements. What the observations actually show is that there is a discrepancy with the Canonical Model, but its origin is uncertain.

* Presented at the XXXIX International Conference of Theoretical Physics "Matter to the Deepest", Ustroń, Poland, September 13-18, 2015. 
At present, there are at least two ways of accounting for the discrepancy without assuming the accelerated expansion: (i) since the spacetime of the real universe is different from the $\mathrm{R}-\mathrm{W}$ one and assuming large scale inhomogeneities, in the Lemaître-Tolman spacetime a toy model has been constructed, which may account for the supernovae type Ia observations without invoking the global acceleration; (ii) the influence of small scale inhomogeneities (galaxies and their groups) is controversial since one research group claims that the effect (the "backreaction") is significant, while another group denies the effect. The polemic is sharp and the problem remains open.

Most researchers in cosmology accept the conservative, traditional approach: the universe is very well fitted by the $\mathrm{R}-\mathrm{W}$ geometry and the cosmic matter is almost uniformly distributed (the backreaction is negligible). Then, the supernovae observations must be interpreted as an accelerated expansion and there are three ways of generating this effect.

(i) The most popular is the concordant $\Lambda \mathrm{CDM}$ model, which is in good conformity with all the observations (Planck satellite, 2013). It is phenomenologically fully satisfactory and conceptually unsatisfactory since it requires a "fine tuning" of the value of $\Lambda$ to the present value of matter (baryonic and dark) density; furthermore, it is extremely difficult to calculate the observationally preferred tiny value of $\Lambda$ from the first principles (i.e. in terms of $\hbar, c$ and $G$ ).

(ii) Dark energy ("quintessence"). The acceleration is driven by a dynamical classical field viewed as a perfect fluid with energy density $\rho_{X}$ and pressure $p_{X}$ satisfying $p_{X} \approx-\rho_{X}<0$. Here most fantastic concepts, like Chaplygin gas, dominate.

(iii) A distinct theory of gravity giving rise to a different dynamics for $a(t)$, then $\Lambda$ and dark energy are redundant. Two questions immediately arise:

- is it possible to replace Einstein's GR, "the most beautiful theory of physics" (Lev Landau) by a better theory?

— is it at all worth doing?

\section{Multitude of theories of gravitation}

We answer the first question. Physics of gravitation is exceptional since in all other branches of physics there is one or at most a couple of competing theories. Yet for gravitation, there is an infinite number of existing or potential theories and GR is, in a sense, just a point in a continuous space 
of conceivable theories. GR is distinguished in this space as a canonical theory: it is perfect since nothing can be removed from it without destroying its structure and falling into conflict with the experiment, also its field equations are the simplest possible. On the other hand, it may be supplemented and expanded without limits by arbitrary new structures and concepts. All these "alternative gravity theories" are merely various modifications, generalizations and always complications of Einstein's GR and they would never arise without it.

These "variations of GR" may be viewed seriously due to scarcity and small variety of experiments and observations in gravitation. Just make a comparison with electromagnetism: one cannot produce gravitational effects (strong fields, black holes, etc.) in such a number and variety as one can in electromagnetism. One does not rule over gravitation as can do over electromagnetic effects. In consequence, many gravity theories fit sufficiently well the accessible empirical data, though in all known tests GR is invariably the best one.

The alternative theories that are most interesting to cosmologists are metric nonlinear gravity (NLG) theories; they differ from GR only in field equations, the Einstein field equations (EFE) are replaced by different ones, also arising from a Lagrangian. Gravity is described by one unifying tensor field $g_{\mu \nu}$, which in the initial formulation of each theory in this class is interpreted as a spacetime metric with a Lagrangian $L=f\left(g_{\alpha \beta}, R_{\alpha \beta \mu \nu}\right)$, where $f$ is any smooth scalar function of the invariants of the curvature tensor. The simplest in this class and most investigated are restricted NLG theories with $L=f(R)$. These theories are sometimes described as "curvature quintessence scenario". NLG theories appeared as first modifications of GR very early (Hermann Weyl circa 1919). They had various motivations: avoidance singularities, better quantization of gravitation; all these hopes failed.

The second question may be reformulated as follows: why to employ the alternative theories instead of assuming that the cosmological acceleration is driven in GR by a kind of self-interacting scalar (vector or tensor) matter field? The answer is that one must then introduce ad hoc some classical field unknown to laboratory physics. All known species of matter exist as quanta of quantized fields and are contained in the Standard Particle Model, yet dark energy is a classical field which does not fit the model and it contradicts the tenet of modern physics that all matter fields are quantized. On the other hand, the gravitational field is classical and need not be quantized (its quantum effects are not known), it is independent of the Standard Model. Replacement of GR by the NLG theory is more conservative than the concept of dark energy. 


\section{Search for a better theory of gravitation}

The leading idea is that if the first real trouble to GR has appeared in cosmology, then the cosmological data should be crucial for recognizing the correct theory. The idea treats the search purely phenomenologically because if observations will point to a specific $L=f\left(g_{\alpha \beta}, R_{\alpha \beta \mu \nu}\right)$, then the question "why this $f$ ?" is postponed to further research in future. The clear weakness of this approach is that NLG theories are applied to and tested only in the spatially flat $\mathrm{R}-\mathrm{W}$ spacetime (solar system tests are purely auxiliary and actually are ambiguous). In this case, the field equations of any theory reduce to one quasi-linear third order ODE for $a(t)$, a quasi-Friedmannian equation (QFE). In vacuum, the Friedmann equation of GR, $\dot{a}^{2}=0$, has only Minkowski spacetime as a solution, whereas NLG theories admit nontrivial solutions. NLG cosmology is, therefore, based on a simplifying assumption that, at present epoch, the higher order terms in QFE dominate over matter contributions and a vacuum solution $a(t)$ accounts for the acceleration. The typical research programme is to seek for $L=f\left(g_{\alpha \beta}, R_{\alpha \beta \mu \nu}\right)$ such that it admits a vacuum solution $a(t)$ yielding the sequence inflation $\Rightarrow$ deceleration $\Rightarrow$ acceleration. For restricted NLG theories, the QFE reads

$$
6\left[\frac{1}{a} \frac{d^{3}}{d t^{3}} a+\frac{\ddot{a} \dot{a}}{a^{2}}-2\left(\frac{\dot{a}}{a}\right)^{3}\right] \frac{\dot{a}}{a} f^{\prime \prime}(R)-\frac{\ddot{a}}{a} f^{\prime}(R)+\frac{1}{6} f(R)=0,
$$

where $f^{\prime}(R)=d f / d R$ with substitution $R=6\left(\ddot{a} / a+\dot{a}^{2} / a^{2}\right)$ and for full NLG theories it is more involved. Only approximate solutions are accessible under an arbitrary assumption that one seeks a solution for which some terms in (1) are negligible. It is rather a little surprise that a great multitude of Lagrangians $L=f(R)$ do fit observational data, these include rational functions, irrational functions, $\exp (c R)$, a combination of two confluent hypergeometric functions. Also

$$
L=R+\frac{1}{R_{\alpha \beta} R^{\alpha \beta}}+\frac{1}{R_{\alpha \beta \mu \nu} R^{\alpha \beta \mu \nu}}
$$

fits the supernovae Ia data.

\section{Criticism of the cosmological search of the correct theory}

In our opinion, this research programme, though suggestive, is actually misleading and hopeless. Our main thesis is that applying cosmological observations is the most unreliable way for recovering the true theory of gravitation. We formulate our criticism in four items. 
1. Most researchers seem to be unaware of the wealth and variety of possibilities. The Lagrangian of a generic NLG theory depends on all 14 invariants of Riemann tensor, $L=f\left(R, R_{\alpha \beta} R^{\alpha \beta}, R_{\alpha \beta \mu \nu} R^{\alpha \beta \mu \nu}, \ldots\right)$. In the simplest case of $L=f(R)$, one invokes a corollary of the Cantor's theorem in set theory stating that the cardinality number of the set of smooth functions $f(R)$ is equal to continuum. In other words, the set of all NLG theories is the continuum. There is rather a little hope to determine a function of 14 variables merely from cosmological observations.

2. In GR, the field equations are of second order, yet in NLG theories in their initial formulation the field equations are of fourth order. However, contrary to a popular prejudice, these are not inherently "higher derivative gravity" theories. Applying appropriate Legendre transformations, one decomposes the initial field $g_{\mu \nu}$ into a multiplet (in general, it is a triplet) of gravitational fields out of which only one is identified with the spacetime metric. The field equations are then of second order and the dynamics of the full multiplet is different from that in GR. The gravitational field in GR has two degrees of freedom, whereas in NLG theories, it has 8 degrees of freedom. The space of solutions is larger than that of GR, also in cosmology. It may contain astonishing solutions, apparently unphysical, and these must be taken into account while evaluating the viability of a given theory.

3. Returning to cosmology, we stress that it tests various gravity theories only in the flat R-W spacetime and its metric is "flexible", it is versatile in that contains an arbitrary function $a(t)$. The QFE (1) for $a$ has solutions for any $f(R)$, i.e. $\mathrm{R}-\mathrm{W}$ metric is a universal solution for all NLG theories, also for a generic Lagrangian. Yet Minkowski, de Sitter and anti-de Sitter spacetimes are in this sense not universal. $\mathrm{R}-\mathrm{W}$ spacetime, its evolution and physical processes in it, is the most inadequate place for discriminating between various NLG theories.

4. If one finds a specific solution fitting the observational cosmology, then it is of little significance even in the framework of the NLG theory to which it belongs. We emphasize the difference in this aspect between GR (including $\Lambda \neq 0$ ) and NLG theories. The standard GR cosmology has the following generic features:

(i) if the cosmic matter satisfies the strong energy condition (SEC), then Hawking-Penrose singularity theorems imply that our universe contains a singularity; 
(ii) in $\mathrm{R}-\mathrm{W}$ spacetime the singularity is in the past (the Big Bang) and $a(t)$ monotonically grows from zero independently of the specific particle content and other properties of the matter (i.e. of the equation of state).

If the SEC does not hold, i.e. for a scalar field, the cosmic evolution may be completely different: no past singularity and there are oscillatory solutions for $t \rightarrow \infty$. In NLG theories, one by assumption neglects the matter, nevertheless a condition equivalent to SEC may be imposed on the nongeometric components of the gravitational multiplet. However, in general, the condition does not hold and the past singularity may not be universal or absent and oscillatory solutions are not excluded. This means that all the successes of the Canonical Cosmological Model are lost and whole cosmology must be constructed anew. The specific solution fitting the observations may not be a generic one in the space of cosmological solutions and it is rather unlikely that the universe evolves according to it.

We now reformulate the fundamental problem of NLG cosmology: suppose that one knows the form of $a(t)$ taken from observations and views it as a solution of some QFE, is it then possible to uniquely and effectively reconstruct the underlying Lagrangian?

We claim that it is impossible. Firstly, because a differential equation is equivalent to the entire space of its solutions and cannot be reconstructed from one or a couple of these. This cannot be done even by a chance when only approximate solutions are accessible. Assume that a Lagrangian $L_{1}$ generates the QFE $F_{1}(a(t))=0$ and the latter has a particular solution $a=a_{1}(t)$ which well describes the cosmic evolution. Let $a_{2}=a_{1}+\delta a(t)$, where $|\delta a / a| \ll 1$ and $\delta a$ is smaller than the observational error bars for the cosmic evolution rate and for the corresponding cosmological effects, then $a_{2}$ equally well fits the data. Furthermore, assume that it can be shown that $a_{2}$ is a solution of another QFE $F_{2}(a)=0$ following from some $L_{2}$. This means that one has two cosmology based theories of gravity, $L_{1}$ and $L_{2}$.

Secondly, the physical interpretation and, in consequence, the experimental verification of any NLG theory is inherently ambiguous. Take the simple case of $L=f(R)$. The field $g_{\mu \nu}$ has 3 degrees of freedom and by assumption it is viewed as the metric. Now, by an appropriate Legendre transformation, it may be decomposed into two fields with definite masses and spins, these are again $g_{\mu \nu}$ viewed as the metric and a scalar $\phi$; the fields are now subject to second order field equations. This is, however, not the end of the story. As it was noticed long ago by Wolfgang Pauli, in the system $\left\{g_{\mu \nu}, \phi\right\}$, one can perform the transformations $g_{\mu \nu} \rightarrow \bar{g}_{\mu \nu}=F_{1}(\phi) g_{\mu \nu}$ and $\phi \rightarrow \bar{\phi}=F_{2}(\phi)$ with arbitrary scalar functions $F_{1}$ and $F_{2}$ of $\phi$. (For a generic 
NLG theory the freedom of making transformations of the gravitational multiplet is larger.) In particular, $F_{1}$ and $F_{2}$ may be so chosen that $\bar{g}_{\mu \nu}$ and $\bar{\phi}$ satisfy the EFE and Klein-Gordon equation (with a potential) respectively. What is then the genuine physical spacetime metric, the initial $g_{\mu \nu}$ or one of the infinite set of conformally related $\bar{g}_{\mu \nu}$ ? Notice that in GR there is no such freedom of interpretation. Many researchers in the field overlook the freedom and arbitrarily assume that $g_{\mu \nu}$ is physical. Actually, one must apply an independent physical criterion to choose the physical metric and such a criterion was put forward twenty years ago, unfortunately it has not been commonly accepted. Without it, any comparison of observations with a theory is ambiguous and inconclusive.

Is there any way out of this situation? In our opinion, if one believes that Einstein's GR should be improved by modifying its field equations (an NLG theory), then one should provide two criteria. First of them, possibly based on classical field theory, should reduce the infinite set of NLG theories to a narrow class or just a couple of them and these theories according to it would be viable ones. No such criterion is known; a candidate criterion reduces the set to a half, i.e. there still remains infinite number of tenable theories. The other criterion should establish which tensor $g_{\mu \nu}$ out of the set of conformally related ones is the physical spacetime metric. As mentioned above, such criterion does exist, but many researchers reject it.

Due to shortage of space these concepts are presented here in a very concise way. A majority of them have been expounded in great detail in papers [1-4].

\section{REFERENCES}

[1] G. Magnano, L.M. Sokołowski, Phys. Rev. D 50, 5039 (1994) [arXiv:gr-qc/9312008].

[2] L.M. Sokołowski, Class. Quantum Grav. 24, 3391 (2007) [arXiv: gr-qc/0702097v2].

[3] L.M. Sokołowski, Class. Quantum Grav. 24, 3713 (2007) [arXiv: 0707.0942v1 [gr-qc]].

[4] L.M. Sokołowski, Acta Phys. Pol. B 39, 2879 (2008) [arXiv:0810.2554v1 [gr-qc]]. 